\title{
ON PRACTICAL STABILITY OF TIME DELAY SYSTEMS
}

\author{
Z. Lj. Nenadic \\ Washington University in St. Louis, St. Louis, MO 63112, USA \\ D. Lj. Debeljkovic, S. A. Milinkovic \\ University of Belgrade, System Control Group, Karnegijeva 4, \\ 11000 Belgrade, Yugoslavia. E-mail: emilinko@ubbg.etf.bg.ac.yu
}

\begin{abstract}
Paper extends some basic results from the area of finite time and practical stability to linear, time-delay systems. Sufficient conditions of this kind of stability are derived.

\section{Introduction}

Weiss and Infante [1, 2] have introduced various notations of stability over finite time interval for continuoustime systems and constant set trajectory bounds. Further development of these results were due to many other authors.
\end{abstract}

\section{Preliminaries}

A linear, multivariable time-delay system can be represented by differential equation:

$$
\dot{\mathbf{x}}(t)=A_{0} \mathbf{x}(t)+A_{1} \mathbf{x}(t-\tau)
$$

and with associated function of initial state:

$$
\mathbf{x}(t)=\Psi_{x}(t), \quad-\tau \leq t \leq 0
$$

Equation (1) is refered to as homogenous or the unforced state equation, $\mathbf{x}(t)$ is the state vector, $A_{0}$ and $A_{1}$ are constant system matrices of appropriate dimensions, and $\tau$ is pure time delay, $\tau=$ const. $(\tau>0)$.

Dynamical behavior of autonomous system (1) is defined over time interval $J=\left\{t_{0}, t_{0}+T\right\}$, where quantity $T$ may be either a positive real number or symbol $+\infty$, so finite time stability and practical stability can be treated simultaneously. It is obvious that $J \in R$.

Let index $\beta$ stands for the set of all allowable states of system and index $\alpha$ for the set of all initial states of the system, such that the set $S_{\alpha} \subseteq S_{\beta}$. In general, one may write:

$$
S_{\rho}=\left\{\mathbf{x}:\|\mathbf{x}(t)\|_{\mathrm{Q}}^{2}<\rho\right\},
$$

where $Q$ will be assumed to be symmetric, positive-definite, real matrix.

\section{Main results}

Definition 1: System given by (1) satisfying initial condition (2) is practically stable w.r.t. $[\zeta(t), \beta, T]$ if and only if:

$$
\Psi_{x}^{T}(t) \Psi_{x}(t)<\zeta(t), \quad \forall t \in[-\tau, 0]
$$

implies:

$$
\mathbf{x}^{T}(t) \mathbf{x}(t)<\beta, \quad \forall t \in[0, T]
$$

$\zeta(t)$ being scalar function with the property $0<\zeta(t) \leq \alpha$, $-\tau \leq t \leq 0$, where $\alpha$ is a real positive number and $\beta \in R$ and $\beta>\alpha$.

Theorem 1: The system given by (1) with the initial function (2) is finite time stable w.r.t. $\{\alpha, \beta, \tau, T\}$, if the following condition is satisfied:

$$
\|\Phi(t)\|<\frac{\sqrt{\beta / \alpha}}{1+\tau\left\|A_{1}\right\|}, \quad \forall t \in[0, T]
$$

where $\|()$.$\| denotes Euclidean norm, and \mathrm{F}(t)$ is fundamental matrix of systeem (1).

Proof. The solution of (1) with initial function (2) can be expressed in terms of fundamental matrix as it is written below:

$$
\mathbf{x}(t)=\Phi(t) \Psi_{x}(0)+\int_{-\tau}^{0} \Phi(t-\theta-\tau) A_{1} \psi_{x}(\theta) d \theta
$$

Using the above equation and the following abbreviations:

$$
\begin{aligned}
& \Phi(t) \psi_{x}(0)=\mathbf{a}(t) \in R^{n \times 1}, \\
& \Phi(t-\theta-\tau) A_{1} \Psi_{x}(\theta)=\mathbf{b}(t, \theta) \in R^{n \times 1}, \\
& \int_{-\tau}^{0} \mathbf{b}(t, \theta) d \theta=\mathbf{c}(t) \in R^{n \times 1},
\end{aligned}
$$

one can get:

$$
\begin{aligned}
\mathbf{x}^{T}(t) \mathbf{x}(t) & =\Psi_{x}^{T}(0) \Phi^{T}(t) \Phi(t) \Psi_{x}(0) \\
& +\mathbf{a}^{T}(t) \mathbf{c}(t)+\mathbf{c}^{T}(t) \mathbf{a}(t)+\mathbf{c}^{T}(t) \mathbf{c}(t)
\end{aligned}
$$

The very well-known result from the theory of quadratic forms gives: 


$$
\Psi_{x}^{T}(0)\left[\Phi^{T}(t) \Phi(t)\right] \Psi_{x}(0) \leq \lambda_{M}(t) \Psi_{x}^{T}(0) \Psi_{x}(0)
$$

where $\lambda_{M}(t)=\max \sigma\left\{\Phi^{T}(t) \Phi(t)\right\}, \mathbf{a}^{T}(t) \mathbf{c}(t)=\mathbf{f}(t) \in R^{1}$ and $\mathbf{c}^{T}(t) \mathbf{a}(t)=\mathbf{f}(t) \in R^{1}$, and it follows that: $\mathbf{a}^{T}(t) \mathbf{c}(t)=\mathbf{c}^{T}(t) \mathbf{a}(t)$. Now, one can write:

$$
\mathbf{x}^{T}(t) \mathbf{x}(t) \leq \lambda_{M}(t) \Psi_{x}^{T}(0) \Psi_{x}(0)+2 \mathbf{a}^{T}(t) \mathbf{c}(t)+\|\mathbf{c}(t)\|^{2}
$$

Moreover:

$$
f(t) \leq|f(t)|, \quad\left|\int_{a}^{b} \varphi(x) d x\right| \leq \int_{a}^{b}|\varphi(x)| d x,
$$

it follows:

$$
\mathbf{a}^{T}(t) \mathbf{c}(t) \leq\left\|\psi_{x}^{T}(0)\right\| \cdot\left\|\Phi^{T}(t)\right\| \cdot \int_{-\tau}^{0}\|\mathbf{b}(t, \theta)\| d \theta .
$$

However, if:

$$
m(t) \leq\|\Phi(t-\theta-\tau)\|\left\|\Psi_{x}(\theta)\right\| \leq M(t), \quad \forall \theta \in[-\tau, 0],
$$

then:

$$
m(t) \tau \leq \int_{-\tau}^{0}\|\Phi(t-\theta-\tau)\|\left\|\Psi_{x}(\theta)\right\| d \theta \leq M(t) \tau .
$$

It is easy to show that:

$$
\begin{aligned}
& \|\Phi(t-\theta-\tau)\|\left\|_{\theta \in[-\tau, 0]} \leq\right\| \Phi(t)\|,\| \Psi_{x}(\theta) \|\left.\right|_{\theta \in[-\tau, 0]}<\sqrt{\alpha} \\
& \lambda_{M}(t) \leq\left\|\Phi^{T}(t) \Phi(t)\right\| \leq\left\|\Phi^{T}(t)\right\|\|\Phi(t)\|=\|\Phi(t)\|^{2}
\end{aligned}
$$

so, it follows from (9):

$$
\begin{aligned}
& \mathbf{x}^{T}(t) \mathbf{x}(t) \leq\|\Phi(t)\|^{2} \Psi_{x}^{T}(0) \Psi_{x}(0) \\
& +2\left\|\psi_{x}^{T}(0)\right\|\left\|\Phi^{T}(t)\right\|^{2}\left\|A_{1}\right\| \tau \sqrt{\alpha}+\left\|\Phi^{T}(t)\right\|^{2}\left\|A_{1}\right\|^{2} \tau^{2} \alpha .
\end{aligned}
$$

If one use (4), then immediately follows:

$$
\begin{aligned}
\mathbf{x}^{T}(t) \mathbf{x}(t) & \leq\|\Phi(t)\|^{2} \alpha+2\|\Phi(t)\|^{2}\left\|A_{1}\right\| \tau \alpha \\
& +\|\Phi(t)\|^{2}\left\|A_{1}\right\|^{2} \tau^{2} \alpha=\|\Phi(t)\|^{2} \alpha\left(1+\tau\left\|A_{1}\right\|\right)^{2} .
\end{aligned}
$$

Applying the basic condition of theorem, equation (6), on the preceding inequality, one can get:

$$
\mathbf{x}^{T}(t) \mathbf{x}(t)<\left(\frac{\sqrt{\beta / \alpha}}{1+\tau\left\|A_{1}\right\|}\right)^{2} \alpha\left(1+\tau\left\|A_{1}\right\|\right)^{2}<\beta,
$$

what has to be proved. When $\tau=0$, or $\left\|A_{1}\right\|=0$, the problem is reduced to the known case of the ordinary linear systems.

Non-delay application of this theorem is in some manner difficult, since one has to find fundamental matrix $\Phi(t)$. In order to overcome this problem, the following discussion is presented.

It is possible to establish the following connection between $\Phi(s)$ and $\Phi_{0}(s)=\left(s I-A_{0}\right)^{-1}$ so, one can write:

$$
\begin{aligned}
\Phi(s) & =\left(I-\Phi_{0}(s) A_{1} e^{-\tau s}\right)^{-1} \Phi_{0}(s) \\
& =\Phi_{0}(s)+\sum_{k=1}^{\infty} \Phi_{0}^{k}(s) A_{1}^{k} e^{-k \pi} \Phi_{0}(s) .
\end{aligned}
$$

Having in mind this discussion, and numerical aspect of computation of matrix $\mathrm{F}(s)$ and $\mathrm{F}_{0}(s)$ [3], the following results can be stated.

Theorem 2: Suppose $\left\|\Phi_{0}(t)\right\|>\|\Phi(t)\| \forall t \in[0, T]$, where matrices $\Phi_{0}(s)$ and $\Phi(s)$ are defined in (21). Then, the system given by (1) with initial function (2) is finite time stable with respect to $\{\alpha, \beta, \tau, T\}$ if the following condition is satisfied:

$$
\left\|\Phi_{0}(t)\right\|<\frac{\sqrt{\beta / \alpha}}{1+\tau\left\|A_{1}\right\|}, \quad \forall t \in[0, T] .
$$

Theorem 3: Suppose $\left\|\Phi_{0}(t)\right\|<\|\Phi(t)\| \forall t \in[0, \mathrm{~T}]$, where matrices $\Phi_{0}(s)$ and $\Phi(s)$ are defined in (21). Then, the system given by (1) with initial function (2) is not finite time stable, if there exists a moment $t=t^{*}$ such that the following inequality is satisfied:

$$
\left\|\Phi_{0}\left(t^{*}\right)\right\|>\frac{\sqrt{\beta / \alpha}}{1+\tau\left\|A_{1}\right\|}, \quad t^{*} \in[0, T] .
$$

The proofs of both theorems follow directly from proof of Theorem 1.

\section{Conclusion}

In the circumstances when it is possible to establish the suitable connection between fundamental matrices of linear time-delay and non-delay systems, presented results enable an efficient procedure for testing finite time stability characteristics of particular class of time delay systems.

\section{References}

[1] Weiss, L., E. F. Infante, "On the Stability of Systems Defined over Finite Time Interval", Proc. National Acad. Science, 54 (1) (1965) 44-48.

[2] Weiss, L., E. F. Infante, "Finite Time Stability under Perturbing Forces on Product Spaces", IEEE Trans. on Automat. Cont., AC-12 (1) (1967) 54-59.

[3] Koepcke, R. W., "On the Control of Linear Systems with Pure Time Delay", Trans. ASME J. of Basic Eng., (3) (1965) 74-79. 